CHRISTINE NOËL ÉTIENNE REDOR

Audencia Nantes École de management

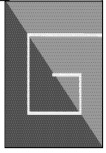

\title{
Les mécanismes de défense anti-acquisition profitent-ils vraiment aux actionnaires?
}

Cet article entreprend de présenter les principaux mécanismes de défense anti-acquisition en questionnant leur intérêt pour les actionnaires et les dirigeants, à partir d'une revue de la littérature. En confrontant la théorie des intérêts des actionnaires et la théorie de l'enracinement, les auteurs montrent que la plupart des mécanismes de défense utilisés profitent davantage aux dirigeants, car ils leur permettent de consolider leur pouvoir et leurs avantages personnels. Les conclusions de cet article sont ainsi propres à renforcer la vigilance des actionnaires car les mécanismes de défense seraient souvent destructeurs de richesse. 
L 'opposition entre la finance, d'une part, et le management, d'autre part, est un préjugé classique qu'il n'est pas aisé de dissiper. Ce préjugé, encore renforcé par la crise financière, alimente plusieurs mythes construits autour des divergences d'intérêts entre les actionnaires et les autres parties prenantes de l'entreprise. Parmi ces mythes, Albouy (1993) mentionne, notamment, l'idée selon laquelle « les OPA mettent en danger la vie de l'entreprise », ce qui justifierait l'utilisation de mécanismes de défense souvent coûteux.

Deux écoles de pensée s'opposent à ce sujet: la théorie des intérêts des actionnaires et la théorie de l'enracinement. Selon la théorie de l'intérêt des actionnaires formulée par DeAngelo et Rice (1983), les actionnaires actuels sont inefficaces pour obtenir une prime plus élevée pour leurs actions $^{1}$. En effet, chaque actionnaire, animé par des motivations privées, est individuellement incapable d'obtenir le prix le plus élevé pour ses actions, ce qui réduit la prime collective des actionnaires en tant que groupe. En agissant dans l'intérêt des actionnaires non coopérants, la direction pourrait être capable d'extraire un prix plus élevé d'un acquéreur potentiel.

Au contraire, selon la théorie de l'enracinement, un dirigeant peut adopter un mécanisme de défense anti-acquisition pour conserver ses avantages personnels. Il cherchera avant tout à conserver son emploi et le niveau de sa rémunération. Ainsi pour Scherer (1988), la menace d'une acquisition et la possible perte d'emploi associée pour le dirigeant peuvent l'inciter à adopter une politique à court terme. De même, selon Stein (1988), la pression des acquisitions est néfaste pour les actionnaires car cela incite les dirigeants à renoncer à des projets profitables à long terme et à concentrer sur des projets moins profitables à court terme dans le but d'augmenter les profits courants. Or, les intérêts des dirigeants et des actionnaires de la société cible coïncident rarement. Pour un actionnaire diversifié, une offre représente simplement l'opportunité de réaliser des gains grâce à la prime. Pour un dirigeant, cela est synonyme soit d'une perte d'emploi, soit d'une diminution de prestige et de sécurité. De ce fait, les dirigeants pourraient décider de résister à une tentative d'acquisition qui aurait eu comme conséquence d'augmenter la richesse de leurs actionnaires (Buchholtz et Ribbens, 1994).

La question de l'impact des mécanismes de défense anti-acquisition sur la richesse des actionnaires a fait l'objet de vifs débats dans le passé. La question se pose alors de l'usage qui peut être fait de ces mécanismes de défense anti-acquisition. Les dirigeants utilisent-ils toujours ces armes juridiques et financières au profit des actionnaires ou au contraire les utilisent-ils pour des intérêts plus personnels? Les études empiriques menées sur la pertinence des mécanismes de défense anti-acquisition pour les actionnaires apportent des résultats contradictoires, ce qui ne permet pas de trancher entre ces deux écoles de pensée ${ }^{2}$. Jarrell et

1. Afin d'inciter les actionnaires de la société cible à participer à l'offre, la société initiatrice propose en général de racheter les actions détenues par les actionnaires de la société cible avec une prime par rapport à leur valeur actuelle. Cette prime est alors appelée la prime d'acquisition. Selon Schwert (1996), elle est de l'ordre de $30 \%$ et selon Officer (2003), elle atteint $45 \%$ en moyenne lors des acquisitions de sociétés cotées.

2. D'un côté, DeAngelo et Rice (1983) obtiennent des pertes non significatives à l'annonce de l'adoption d'un mécanisme de défense anti-acquisition et interprètent ce résultat comme une faible preuve validant l'hypothèse d'enracinement et de l'autre, Linn et McConnell (1983) mettent en évidence que l'adoption de ces mêmes mécanismes est associée à une réaction positive du marché, validant ainsi la théorie de l'intérêt des actionnaires. 
Poulsen (1987) montrent que les différents mécanismes ont des impacts distincts sur la richesse des actionnaires. Certains mécanismes favoriseraient plutôt le comportement d'enracinement tandis que d'autres favoriseraient l'intérêt des actionnaires.

L'objectif de cet article est de présenter les principaux mécanismes de défense antiacquisition en évaluant dans quelle mesure ceux-ci permettent de concilier l'intérêt des actionnaires et celui des dirigeants, au regard de la théorie de l'intérêt des actionnaires et de la théorie de l'enracinement. Nous mettons en évidence, à partir d'une analyse juridique des mécanismes existants et d'une revue de la littérature, le fait que ces mécanismes de défense profitent davantage aux dirigeants, leur permettant de consolider leur position et leurs avantages au sein de la société. Ces éléments sont ainsi propres à renforcer la vigilance des actionnaires dans la mesure où les mécanismes de défense seraient souvent destructeurs de richesse.

Notre démarche se décline en trois temps. La première section s'intéresse aux défenses antérieures à l'offre d'acquisition. La seconde section examine les mécanismes de défense postérieurs à l'offre d'acquisition. Enfin, la dernière section s'intéresse à l'utilisation conjointe de mécanismes de défense et d'un parachute doré.

\section{I - LES MÉCANISMES DE DÉFENSE ANTÉRIEURS À L'OFFRE}

Il existe un certain nombre de dispositifs anti-acquisitions qui peuvent être mis en place par les sociétés avant qu'une offre ne soit réalisée par la société initiatrice. Il s'agit de la super-majorité, de l'échelonne- ment des élections du conseil d'administration, du prix juste et enfin des pilules empoisonnées.

\section{La super-majorité}

Ce dispositif augmente le nombre de droits de votes nécessaires pour approuver une fusion. Dans ce cas, le seuil à atteindre est souvent de $80 \%$, mais il peut parfois aller jusqu'à $95 \%$. En général, la super-majorité nécessaire pour approuver une prise de contrôle est accompagnée par un mécanisme de verrouillage. Une super-majorité est alors également nécessaire pour modifier les dispositifs anti-acquisitions. Une minorité d'actionnaires se voit ainsi pourvus d'un droit de veto par lequel ils peuvent bloquer une fusion. En augmentant le coût d'une offre hostile, la super-majorité encourage les acquéreurs potentiels à négocier avec le conseil d'administration de la société cible. En effet, le conseil d'administration se réserve en général la possibilité de renoncer à cette disposition si la majorité des administrateurs approuve la fusion.

Le dispositif de la super-majorité permet ainsi aux dirigeants de faire échouer une offre qui n'est pas dans l'intérêt des actionnaires. Mais ce dispositif leur permet également de s'assurer que l'offre sera suffisamment élevée pour être acceptée par les actionnaires ou de s'enraciner contre une offre à laquelle ils s'opposent. Les études empiriques consacrées à l'usage qu'il est fait de la super-majorité semblent plutôt confirmer la dernière hypothèse. DeAngelo et Rice (1983), Linn et McConnell (1983) puis Mahoney et Mahoney (1993) mettent en évidence les effets négatifs de ce dispositif sur les cours. De plus, Jarrell et Poulsen (1987) montrent que les dispositifs de super-majorité assortis de clauses déro- 
gatoires sont associés à des pertes statistiquement significatives de $-5 \%$.

Au contraire, les dispositifs de super-majorité sans clauses dérogatoires sont associés à des pertes non significatives de $-1 \%$. Selon ces résultats, le pouvoir discrétionnaire par lequel les dirigeants se réservent la possibilité de renoncer au dispositif de la super-majorité ne contribuerait pas à la création de richesse.

\section{L'échelonnement des élections du conseil d'administration}

Selon cette disposition, le conseil d'administration est divisé en trois groupes. Chaque année seule un groupe, ou un tiers des administrateurs est élu. Ceci rend donc difficile pour un acquéreur hostile d'obtenir le contrôle immédiat de la société cible, même s'il possède une majorité des actions. Ce dispositif est souvent accompagné par un mécanisme prévoyant que l'accord de super-majorité par les actionnaires est requis pour changer le nombre d'administrateurs. Ainsi l'acquéreur ne pourra pas agrandir le conseil d'administration afin de le contrôler en élisant des alliés aux nouveaux postes (Mahoney et al., 1997).

L'échelonnement des élections du conseil d'administration est fréquemment justifié par la direction en termes de continuité: la société disposant d'un tel mécanisme n'aura jamais un conseil d'administration composé d'une majorité de nouveaux membres non expérimentés. Cependant, cet avantage peut également se transformer en inconvénient. Bien que selon Faleye (2006), l'échelonnement des élections du conseil d'administration n'a pas d'impact sur la rotation des administrateurs, il peut malgré tout favoriser un enracinement du conseil d'administration, ce qui pourrait diminuer significativement l'efficacité de la gouvernance (Sundaramurthy et al., 1996).

L'utilité de ce dispositif pour les actionnaires est l'objet de résultats contradictoires. Jarrell et Poulsen (1987) mettent en évidence des gains négatifs mais non significatifs de $1 \%$ lors de l'adoption de cette mesure. En outre, DeAngelo et Rice (1983) et Linn et McConnell (1983) ne trouvent aucun effet sur le cours au moment de la date d'envoi des convocations des actionnaires. Mais ils mettent en évidence des gains au cours de la période allant de la date de convocation à la date de l'assemblée des actionnaires, ce qui tendrait à confirmer l'utilité de ce dispositif pour les actionnaires. À l'inverse, Mahoney et Mahoney (1993) trouvent des rentabilités anormales négatives significatives pour un échantillon de 192 opérations.

De même, Bebchuk et Cohen (2005) étudient l'effet des élections échelonnées sur la valeur des sociétés mesurées par le q de Tobin. Ils montrent que cette disposition est associée à une diminution significative de la valeur de la société. Par ailleurs, Siegel et Karim (1998) montrent que les dirigeants achètent des actions de la société avant d'adopter cette disposition lorsqu'une acquisition est imminente afin de toucher la prime. Au contraire, ils n'achètent pas d'actions lorsqu'ils ne disposent pas d'information concernant la probabilité d'une acquisition. Selon ces auteurs, la motivation principale à l'origine de cette disposition est donc l'enracinement. De même, les travaux de Faleye (2006) révèlent que l'échelonnement des conseils d'administration bénéficie principalement à la direction au détriment des actionnaires car ce mécanisme protège significativement la direction de la discipline du marché. En particulier, il 
montre que ce dispositif réduit la probabilité d'un remplacement forcé de P-DG et est lié à une sensibilité plus faible de la rémunération du P-DG aux modifications de richesse des actionnaires.

Les résultats concernant l'effet dissuasif de ce mécanisme sont également contradictoires. Alors que Pound (1987) met en évidence une plus faible activité d'acquisition entre 1974 et 1984 pour les sociétés ayant adopté à la fois la super-majorité et les élections échelonnées, Ambrose et Megginson (1992) montrent que la probabilité des acquisitions entre 1981 et 1986 n'est pas significativement corrélée avec le fait que la société procède à des élections échelonnées. Enfin, dans la lignée des travaux de Pound (1987), Bebschuk et al. (2002) montrent qu'une telle disposition double la probabilité que la cible d'une opération hostile demeure indépendante. Ces auteurs montrent également que l'échelonnement du conseil d'administration ne se traduit pas par des primes plus élevées si la société cible est acquise, ce qui contredit la théorie de l'intérêt des actionnaires.

\section{La disposition de prix juste}

Cette disposition nécessite qu'un offreur propose un prix juste. Le prix juste est défini soit comme le prix le plus élevé payé par l'offreur pour les actions de la société cible qu'il a déjà acquis, soit comme le prix approuvé par le conseil d'administration de la société cible. Le non-respect par l'acquéreur de cette règle déclenche une disposition de super-majorité. Ce mécanisme de défense est utilisé pour éviter les offres d'acquisitions à deux étages. Dans de telles offres, la société initiatrice fait une offre publique avec un prix raisonnable afin d'obtenir une participation qui lui offrirait le contrôle de la société (le premier étage). Généralement, le deuxième étage offre un prix de fusion substantiellement plus faible que le prix proposé pour le premier étage. Ceci incite les actionnaires à apporter leurs actions à l'offre du premier étage en vue d'obtenir le prix le plus élevé.

Les résultats empiriques révèlent majoritairement l'intérêt de ce dispositif pour les dirigeants. Jarrell et Poulsen (1987) mettent en évidence des modifications de cours négatives bien que non significatives de - 0,65\% pour les 143 mécanismes de prix justes dans leur échantillon. Borokhovich et al. (1997) montrent que les P-DG de sociétés adoptant une super-majorité et une disposition de prix juste perçoivent des salaires plus élevés que les P-DG de sociétés n'en ayant pas adopté.

\section{Les pilules empoisonnées}

Les pilules empoisonnées sont des plans d'attribution de droits de souscription d'actions privilégiées adoptés par le conseil d'administration ${ }^{3}$. Ces droits sont émis pour les actionnaires et demeurent inactifs tant qu'ils ne sont pas déclenchés. Le déclenchement a lieu lorsqu'une offre publique est réalisée pour une fraction importante de la société (généralement au moins $30 \%$ ) ou après qu'un actionnaire individuel accumule un bloc d'actions

3. Il existe une différence importante entre le dispositif mis en place en France et celui utilisé par les sociétés américaines. En effet, la loi française stipule que les actionnaires devront donner leur accord pour l'émission de bons de souscription d'actions lors d'une offre hostile alors qu'aux États-Unis ces pilules empoisonnées peuvent être émises par le conseil d'administration sans qu'il soit nécessaire que l'actionnaire ne donne son accord. Des sociétés telles que Hermès International et Suez ont déjà fait voter de telles dispositions par leurs actionnaires. 
important (généralement $20 \%$ ). Les droits déclenchés peuvent être rachetés par le conseil d'administration au cours d'une courte période lorsque l'événement déclencheur n'a pas eu lieu. Si les droits ne sont pas rachetés, ils peuvent être exercés. On distingue les plans flip over et les plans flip in. Un plan flip over permet aux actionnaires de la société cible (à l'exception de l'acquéreur) d'acheter des actions de l'acquéreur à une décote substantielle après la fusion afin de rendre excessivement cher le fait de fusionner avec la société cible. Un plan flip in permet aux actionnaires de la société cible (sauf l'acquéreur) d'acheter des actions de la société cible. Le principal avantage du plan flip in est qu'il dilue la participation de la société initiatrice dans la société cible. L'utilisation combinée de ces deux plans est envisageable.

Là encore les résultats empiriques ne sont pas unanimes. Ryngaert (1988) a examiné l'adoption de 283 pilules empoisonnées et obtient des rentabilités anormales significatives de $-0,34 \%$ pour les deux jours autour de l'annonce. Il montre par ailleurs que les formes les plus restrictives de pilules empoisonnées sont associées à des réactions significativement négatives du cours de Bourse. De même, Malatesta et Walking (1988) ont examiné 132 pilules empoisonnées et obtiennent des rentabilités anormales de $-0,92 \%$ très fortement significatives. De plus, ils montrent que les dirigeants dont les sociétés adoptent des pilules empoisonnées ont une participation plus faible dans leur société que la moyenne du secteur. Choi et al. (1989) étudient les pilules empoisonnées entre 1985 et 1986. Sur 267 opérations, ils mettent en évidence une réaction négative de $-0,48 \%$ significative au seuil de $1 \%$ sur la période $(-1,+1)$.
Ces résultats révèlent que l'adoption de pilules empoisonnées est cohérente avec l'hypothèse d'enracinement managérial. Comment et Schwert (1995) montrent qu'une offre d'acquisition antérieure diminue l'effet de l'annonce de la pilule de $-2,09 \%$. Ce résultat confirme les travaux de Ryngaert (1988) qui met en évidence une rentabilité anormale de $-1,51 \%$ pour son groupe de contrôle composé d'opérations ayant déjà une offre d'acquisition en cours. Autrement dit, lorsque la pilule empoisonnée est utilisée lorsque l'offre d'acquisition hostile est déjà annoncée, la réaction du cours est plus négative. Ce résultat peut s'expliquer de deux manières. Soit il traduit l'effet dissuasif de la pilule empoisonnée. Soit il traduit l'intégration par le marché d'une nouvelle négative à propos de la progression des négociations concernant l'acquisition. Néanmoins, Ambrose et Megginson (1992) et Comment et Schwert (1995) fournissent des preuves concernant l'absence de caractère dissuasif des pilules empoisonnées. Malgré tout, Comment et Schwert (1995) montrent que de telles mesures anti-acquisitions sont associées à des primes plus importantes pour les actionnaires de la société cible ce qui indique une hausse possible dans leur pouvoir de négociation contre les acquéreurs. Brickley et al. (1994) montrent que la réaction moyenne du marché aux annonces de pilules empoisonnées est positive lorsque le conseil d'administration a une majorité d'administrateurs externes et négative dans le cas contraire. Ce résultat est conforme à l'idée que les dirigeants peuvent profiter d'un manque de contrôle pour réaliser des opérations qui maximisent leur propre bien-être. En effet, les administrateurs externes sont supposés surveiller 
plus efficacement les dirigeants, ce qui expliquerait la réaction positive lorsque les administrateurs externes sont majoritaires. Datta et Iskandar-Datta (1996) montrent qu'en moyenne les détenteurs d'obligations réalisent des pertes lors de l'annonce d'une pilule empoisonnée, alors que les actionnaires ne sont pas affectés. L'impact négatif sur les détenteurs d'obligations est cohérent avec l'hypothèse que l'adoption d'une pilule empoisonnée pourrait signaler une menace d'acquisition qui se traduit en général par une hausse de l'endettement. Les résultats montrent que plus la hausse de l'endettement de la société après l'adoption est importante, plus les effets contraires sur la richesse des détenteurs d'obligations seront élevés. Ce résultat implique que les détenteurs d'obligations sont capables d'anticiper correctement le degré de hausse d'endettement au moment de l'annonce de l'adoption. Les auteurs montrent également que la proportion de propriété des insiders est négativement liée à la modification de la richesse des détenteurs d'obligations à l'annonce de l'adoption de la pilule. Ceci montre que la propriété plus élevée des insiders conduit à un alignement plus important des intérêts des dirigeants sur ceux des actionnaires tout en augmentant les coûts d'agence entre actionnaires et détenteurs d'obligations.

\section{II - LES MESURES DE DÉFENSE POST-OFFRE}

Après qu'un acquéreur a réalisé une offre publique hostile, les actions défensives incluent la plupart des défenses anti-acqui-

\section{LES BONS «BRETONS »}

La controverse concernant l'usage des pilules empoisonnées avait été ravivée en 2006 en France par la décision du Gouvernement d'autoriser l'émission de bons de souscription d'actions lors d'une offre hostile. L'origine de cette loi est liée à l'offre de Mittal Steel sur Arcelor et plus généralement à la volonté du gouvernement français de ne pas voir les « fleurons de l'industrie française » (Danone, Alcatel, etc.) passer sous un contrôle étranger.

Ces « bons Bretons », du nom du ministre de l'Économie, sont des dispositifs proches de la pilule empoisonnée inventée dans les années 1980 aux États-Unis. Ils sont prévus par l'article L 233-32 du code de commerce. Lors d'un plan de pilule empoisonnée, des bons sont émis pour permettre aux actionnaires d'acquérir des actions de leur société ou de la société initiatrice avec une décote importante dès qu'un acquéreur hostile achète un certain pourcentage des actions en circulation. En cas d'utilisation de la pilule empoisonnée, la société initiatrice voit sa participation dans la société cible diluée ou le coût de l'acquisition fortement augmenté. Ainsi, la pilule empoisonnée constitue une arme dissuasive efficace placée entre les mains des dirigeants.

Ce dispositif est encore peu utilisé pour l'instant. Depuis 2007, $20 \%$ des sociétés du CAC 40, à l'instar de Suez, Pernod-Ricard ou Essilor, ont déposé des projets de résolution concernant l'usage de « bons Bretons ». La décision d'émettre de tels bons relève en effet de la compétence de l'assemblée générale extraordinaire. 
sitions antérieures à l'opération et différents mécanismes utilisés à la suite de l'opération. L'objectif de la prochaine section est de décrire le fonctionnement des mesures de défense post-offre.

\section{Le chevalier blanc}

Le terme de chevalier blanc se réfère en général à un acquéreur potentiel incité par la direction de la société cible à surenchérir sur une offre initiale à laquelle s'oppose la direction de la société cible. Le fait que le chevalier blanc attende une offre initiale et une incitation de la société cible pour faire une offre suggère qu'il a besoin de la coopération de la société cible pour profiter de façon maximale de l'acquisition (Shleifer et Vishny, 1986). Parmi les mécanismes de défense envisageables par les dirigeants des sociétés cibles, le recours à un chevalier blanc est particulièrement intriguant. En effet, contrairement à la plupart des autres moyens de défense qui permettent de conserver l'indépendance de la cible, une défense impliquant l'utilisation d'un chevalier blanc peut se traduire par l'acquisition de la société cible.

En introduisant un nouvel acquéreur potentiel dans la lutte pour le contrôle de la cible, le dispositif du chevalier blanc peut permettre d'obtenir une prime plus élevée, ce qui est dans l'intérêt des actionnaires. Ce dispositif permet également de chercher un acquéreur qui a plus de chance de laisser en place la direction de la société cible. Dès lors, un chevalier blanc est vu comme un secours pour la direction de la société cible qui sert les dirigeants inefficaces ou dont les capacités sont sous-estimées.

\section{Les rachats ciblés}

Ces transactions, communément appelées greenmail, ont lieu lorsqu'une société achète un bloc de ses propres actions détenu par un actionnaire unique ou un groupe d'actionnaires. Ce rachat se fait souvent avec une prime ${ }^{4}$ et l'offre de rachat n'est pas étendue à d'autres actionnaires. Les rachats ciblés peuvent être utilisés comme une méthode de défense en offrant une

\section{UN CHEVALIER BLANC POUR WAVECOM}

Le 6 octobre 2008, la société néerlandaise Gemalto annonce son souhait d'acquérir le spécialiste français des cartes à puces, la société Wavecom, malgré l'opposition forcenée du management de cette dernière. Le 3 décembre 2008, le canadien Sierra Wireless, spécialisé dans les modems de connexion aux réseaux de téléphonie mobile de nouvelle génération, propose au management de Wavecom de jouer le rôle de chevalier blanc. Le conseil d'administration de Wavecom adhère immédiatement à la proposition amicale de la société canadienne qui offre de racheter Wavecom pour 218 millions d'euros, ce qui représente $20 \%$ de mieux que l'offre initiale de Gemalto. Le 18 février 2009, Sierra Wireless annonce le succès de son offre puisqu'elle détient désormais $84,34 \%$ des actions Wavecom et 90,5\% des droits de vote. Cet exemple récent illustre parfaitement l'intérêt que peut revêtir le recours à un chevalier blanc pour les actionnaires de la société cible d'une offre hostile.

4. Klein et Rosenfeld (1988a) montrent que la prime moyenne payée lors des greenmails est $22 \%$. 
récompense à un acquéreur pour qu'il mette un terme à l'offre et qu'il vende ses actions à la société initiatrice avec un bénéfice.

Selon la théorie de l'enracinement, un rachat ciblé d'actions peut éliminer le contrôle effectué par le détenteur de bloc d'actions. Il peut également réduire la probabilité d'une acquisition qui, si elle était menée, pourrait coûter aux dirigeants leur poste. Dans cette optique, les acteurs profitant le plus d'une opération de rachat ciblé sont l'équipe de direction (dont le travail est temporairement préservé) et le détenteur de blocs d'actions qui vend ses actions avec une prime. Les actionnaires qui ne participent pas à l'opération sont au contraire les perdants. D'une part, leur richesse a diminué du fait du transfert de richesse entre actionnaires non participants vers le détenteur du bloc d'actions. D'autre part, la réduction de la menace d'acquisition peut être à l'origine d'une hausse possible du comportement opportuniste du dirigeant. Enfin, une perte de gains liée aux synergies liées à l'acquisition est possible. La pertinence même des rachats ciblés est sujette à questionnement. Dans la mesure où le greenmail protège contre un seul acquéreur, d'autres investisseurs peuvent être incités à copier cette stratégie. Par conséquent, des tentatives additionnelles de greenmail par d'autres raiders potentiels pourraient être attendues (Klein et Rosenfeld, 1988b).

Les études empiriques indiquent que l'impact est significativement négatif à l'annonce du rachat ciblé d'actions (Dann et DeAngelo, 1983; Bradley et Wakeman, 1983; Mikkelson et Ruback, 1985, 1991). Néanmoins, Mikkelson et Ruback (1991), Klein et Rosenfeld (1988b) et Holderness et
Sheehan (1985) montrent que cette perte est plus que compensée par les hausses de cours associées avec l'achat initial du bloc. La réaction négative du cours à l'annonce d'un rachat ciblé semble donc être causée par le renversement des prévisions d'acquisition formées lors de l'investissement initial.

De plus, dans un monde où les dirigeants sont mieux informés que le marché concernant les opportunités d'acquisitions de la société, une réaction négative du marché à un paiement de greenmail est en lui-même insuffisant pour conclure contre l'hypothèse de l'intérêt de l'actionnaire. Par exemple, Shleifer et Vishny (1986) présentent un modèle d'asymétrie informationnelle où le paiement de greenmail augmente la valeur intrinsèque de la société tout en causant une diminution du cours de la société. En effet, selon ces derniers, le fait de décourager certains acquéreurs potentiels pourrait augmenter la richesse des actionnaires car cela en encourage d'autres à poursuivre une combinaison avec la cible. Ceci est possible parce que le nombre d'acquéreurs en compétition est réduit et parce que la résistance peut signaler que la cible n'a pas accès à un chevalier blanc. Dans le cas spécifique d'un greenmail, les dirigeants pourraient augmenter les gains attendus d'une acquisition en achetant la participation d'un acquéreur potentiel, ce qui pourrait inciter les autres à étudier une acquisition.

Les greenmails s'accompagnent généralement d'un accord de statu quo (ou standstill agreement). Ces dispositifs limitent la propriété d'une société donnée pour une période de temps spécifiée. Les résultats empiriques par Dann et DeAngelo (1983) montrent que l'adoption d'un accord de 
statu quo est associée à une diminution significative du cours de $4 \%$. De plus, Mikkelson et Ruback (1991) montrent que les gains négatifs en réponse aux rachats ciblés sont plus grands lorsqu'ils sont accompagnés par des accords de statu quo. Ces accords semblent donc réduire la richesse des actionnaires de la société cible. Mais la diminution de cours pourrait simplement représenter la déception du marché concernant le fait qu'une acquisition n'aura pas lieu. Tout comme pour les rachats d'actions, les gains négatifs pourraient simplement représenter le renversement des prévisions favorables.

\section{Acquisitions et désinvestissements}

Les modifications dans la structure de l'actif de la société peuvent également être utilisées pour se défendre contre une offre d'acquisition. De telles tactiques incluent aussi bien le désinvestissement d'un actif convoité par l'acquéreur, que l'achat d'actifs que l'acquéreur ne veut pas, ou encore l'achat d'actifs qui créerait des problèmes réglementaires notamment au regard de la loi antitrust. Chacune de ces actions rend la société cible moins attractive pour la société initiatrice, et réduit d'autant le prix que l'acquéreur accepte de payer pour la cible. Les données fournies par Dann et DeAngelo (1986) pour des opérations de ce type révèlent une réaction négative mais non significative.

De même, la société peut décider de restructurer son passif. L'émission de titres à droit de vote peut augmenter le nombre d'actions à racheter pour l'acquéreur. En général, la société place ces titres à droit de vote dans des mains amicales qui acceptent de soutenir les dirigeants actuels. Le rachat peut également être utilisé pour réduire le nombre d'actions cotées, ce qui rend plus difficile le fait d'acheter suffisamment d'actions pour obtenir le contrôle. Ces rachats sont souvent financés par des émissions de dettes qui pourraient rendre les sociétés moins attractives pour les sociétés initiatrices potentielles. Ces restructurations semblent avoir un impact négatif sur la richesse des actionnaires. Dann et DeAngelo (1986) mettent en évidence une diminution du cours de $2 \%$ en moyenne, sur un échantillon de 31 restructurations.

\section{Les procès}

Enfin, la défense de post-offre la plus commune est de faire un procès à la société initiatrice. Jarrell (1985) met en évidence que les litiges surviennent dans un tiers des offres publiques réalisées entre 1962 et 1980. Les plaintes peuvent porter sur des fraudes, des violations de lois antitrust... Le dépôt de plainte a deux objectifs. Premièrement, il retarde l'acquéreur, ce qui peut encourager l'entrée d'un nouvel acquéreur dans la lutte pour le contrôle de la société cible. Confirmant cette idée, Jarrell (1985) montre que, dans $62 \%$ des cas, plusieurs acquéreurs luttent pour obtenir le contrôle de la société cible lorsqu'une plainte a été déposée contre simplement $11 \%$ des cas en l'absence de plainte. Deuxièmement, le dépôt de plainte encourage l'acquéreur à augmenter le prix d'offre pour inciter la cible à laisser tomber les poursuites et donc éviter des dépenses de justice. Jarrell (1985) montre pour 71 plaintes que l'effet des poursuites sur le cours boursier est nul en moyenne. 


\section{EN 2008, DES ACTIONNAIRES PLUS ATTENTIFS À L'ADOPTION DE MÉCANISMES ANTI-ACQUISITIONS}

Comme le soulignait Albouy, dès 1996, les dirigeants ont tendance à se protéger des mécanismes de sanction du marché tels que les offres publiques et prennent parfois des décisions non conformes aux intérêts des actionnaires. Néanmoins, il souligne qu'une évolution des comportements est inéluctable. Une telle évolution semble se dégager des assemblées générales tenues en 2008. En effet, les actionnaires se sont révélés particulièrement attentifs à l'égard des projets de résolutions qui leur étaient présentés concernant l'adoption de dispositifs anti-acquisitions. Certaines sociétés, telles que Vallourec, ont ainsi renoncé à l'idée d'émettre des « bons Bretons » par peur de déplaire à leurs actionnaires. En 2008, dix projets de résolution relatifs à l'adoption de mécanismes anti-acquisitions ont finalement été retirés avant la tenue de l'AGE pour des raisons similaires.

Parmi les armes anti-OPA soumises au vote des actionnaires, seuls les rachats d'actions, dont la finalité n'est pas exclusivement de s'opposer à une offre hostile, ont été validés par les actionnaires.

\section{III - L'UTILISATION CONJOINTE DE MÉCANISMES DE DÉFENSE ET DE PARACHUTE DORÉ}

Les parachutes dorés sont des accords contractuels offrant une indemnité importante aux dirigeants qui doivent quitter la société lorsqu'une acquisition a lieu. Les partisans de cette disposition expliquent que les parachutes dorés permettent à la fois d'attirer les meilleurs dirigeants et de faire coïncider les intérêts des dirigeants et des actionnaires en diminuant la probabilité que la direction de la société cible résiste à une offre qui maximiserait la richesse des actionnaires. Les parachutes dorés sont souvent présentés comme une solution au problème de l'enracinement (Baron, 1983; Lambert et Larcker, 1985). En effet, si le dirigeant reçoit un paiement important en cas de perte d'emploi suite à une acquisition, il sera moins enclin à essayer de blo- quer sa réalisation. À l'inverse, les opposants à cette disposition estiment qu'il est du devoir des dirigeants de travailler en vue de maximiser la richesse des actionnaires. Dès lors, il n'existe pas de raisons de leur offrir une récompense particulière pour faire leur travail.

Il n'est pas rare que certains dirigeants de sociétés se voient attribuer des parachutes dorés alors même que leur société dispose d'un ou plusieurs mécanismes de défense. L'utilisation simultanée de mécanismes destinés à réduire la probabilité d'acquisition de la société et de mécanismes permettant au contraire d'augmenter la probabilité de cette même acquisition est surprenante. La question de l'intérêt réel des actionnaires se pose légitimement dans ce cas. Néanmoins, Harris (1990) montre qu'il pourrait être parfaitement rationnel pour les actionnaires de la cible d'adopter à la fois des dispositifs anti- 
acquisitions et de fournir aux dirigeants des parachutes dorés ou d'adopter des dispositions anti-acquisitions seules. En faisant cela, les actionnaires de la société cible peuvent capter une proportion plus importante des gains en synergie qui résulteraient de la combinaison de la cible et de l'initiatrice. De même, Knoeber (1986) développe un cadre conceptuel dans lequel une combinaison de parachutes dorés et de dispositifs anti-acquisitions pourrait être avantageuse. Une offre publique réussie permet ainsi aux nouveaux propriétaires de remplacer la direction et de capter la rémunération différée qui leur est due. Les parachutes dorés et les dispositifs anti-acquisition réduisent la probabilité que ceci arrive et peuvent donc être vus comme des mécanismes adoptés par les actionnaires afin d'assurer aux dirigeants leur intégrité en les incitant à accepter un contrat qui satisfait les deux parties.

Selon Schwartz (1998), lorsque la société dispose d'un mécanisme de défense et que le dirigeant bénéficie d'un parachute doré, ce dernier est incité à obtenir une prime plus élevée tout en acceptant la fusion. Dans le cadre des lois anti-acquisitions adoptées dans différents pays, il montre en effet que les sociétés qui ont mis en place des parachutes dorés réalisent des rentabilités anormales cumulées de l'ordre de $16 \%$.
Au contraire, les sociétés qui n'ont pas mis en place de parachutes dorés réalisent des pertes anormales cumulées de $-7 \%$. Les marchés financiers semblent donc réagir comme si les dirigeants pourvus de parachutes dorés agissaient dans l'intérêt des actionnaires.

Toutefois, Buchholtz et Ribbens (1994) montrent que l'existence de parachutes dorés n'a pas d'effet sur la résistance à une offre. Ce résultat est important car il est contradictoire avec l'idée selon laquelle la réaction positive observée à l'annonce de l'opération signifierait que celle-ci est dans l'intérêt des actionnaires ${ }^{5}$. En effet, puisque l'existence d'un parachute doré n'a pas d'impact sur la résistance de la société cible, la réaction positive du marché peut être interprétée comme la simple prise en compte d'une augmentation de la probabilité d'acquisition. Une autre explication pourrait être que le marché est convaincu que les managers bénéficiant de parachutes dorés ont plus de chance de diriger la société avec une vue à long terme puisqu'ils possèdent des protections contre les modifications du contrôle. Dans tous les cas, les résultats empiriques observés infirment l'idée selon laquelle la réaction positive du cours indiquerait une diminution de la probabilité de résistance.

\footnotetext{
5. Lambert et Larcker (1985) mettent en évidence des réactions positives de cours à l'annonce de l'adoption de parachutes dorés. Néanmoins, comme le souligne Jensen (1988), il n'est pas possible de savoir si cette réaction positive du cours reflète la conviction des investisseurs que les parachutes dorés sont dans l'intérêt des actionnaires ou si les sociétés qui adoptent de tels dispositifs augmentent la probabilité d'être prochainement la cible d'une acquisition. De plus, les travaux de Lambert et Larcker (1985) sont contestés par Born et al. (1993) qui montrent que seules les sociétés non encore engagées dans des acquisitions obtiennent des réactions positives à l'annonce et que les sociétés concernées par des projets d'acquisitions obtiennent des rentabilités nulles. Ce résultat est cohérent avec l'idée que les parachutes dorés sont un signal positif concernant le fait que la société cible a augmenté la probabilité qu'une firme reçoive une offre d'acquisition. Par ailleurs, Mogavero et Toyne (1995) montrent que sur la période 1982-1990, les réactions anormales de cours des sociétés adoptant un parachute doré sont négatives.
} 


\begin{tabular}{|c|c|c|c|c|c|c|c|c|}
\hline 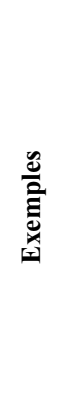 & 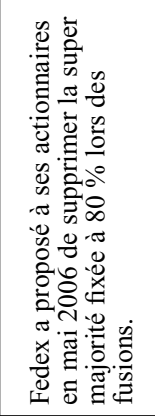 & 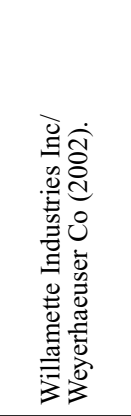 & 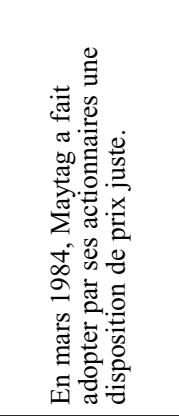 & 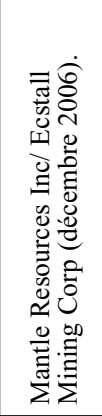 & 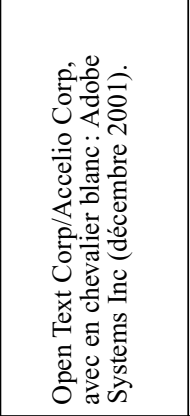 & 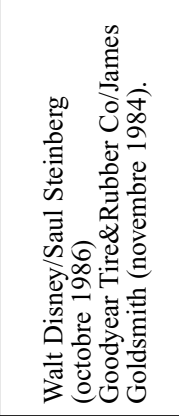 & 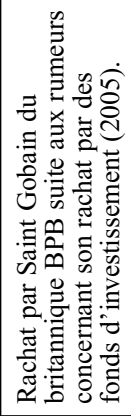 & 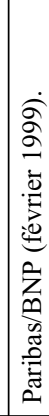 \\
\hline 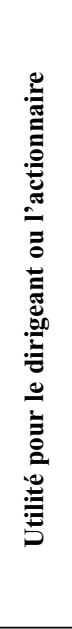 & 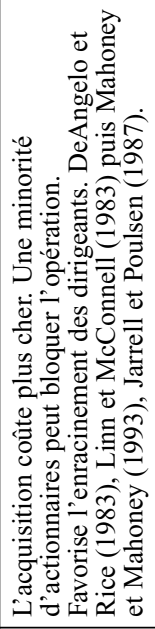 & 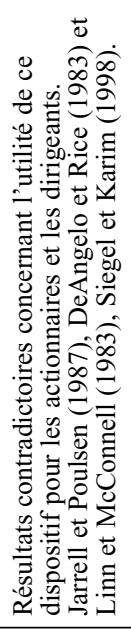 & 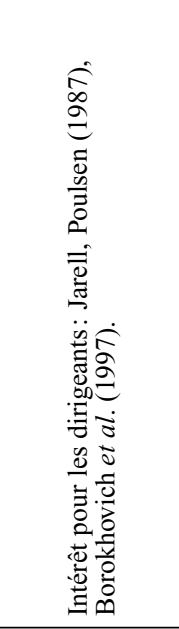 & 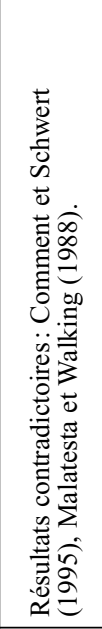 & 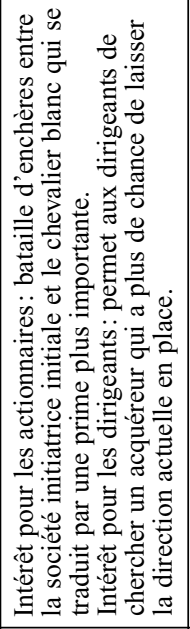 & 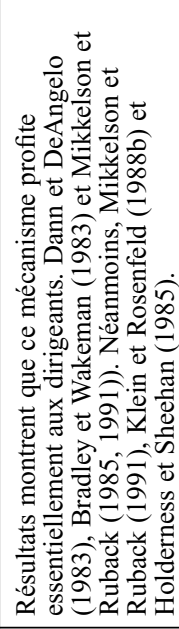 & 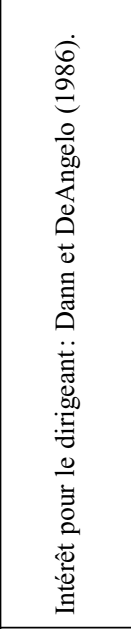 & 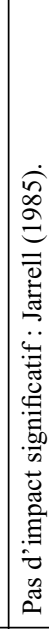 \\
\hline 苛 & 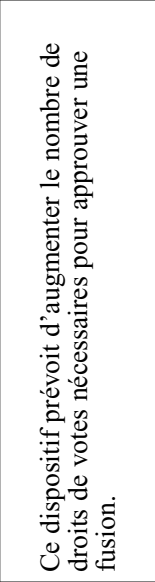 & 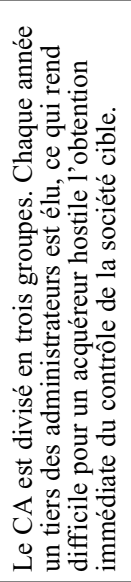 & 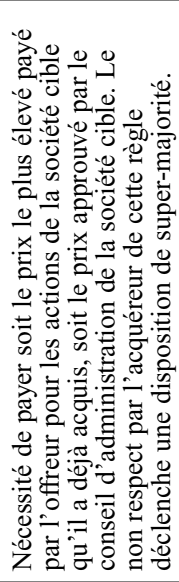 & 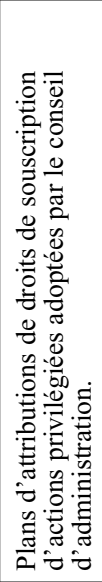 & 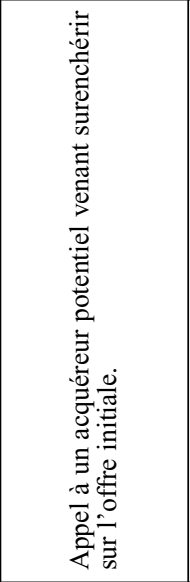 & 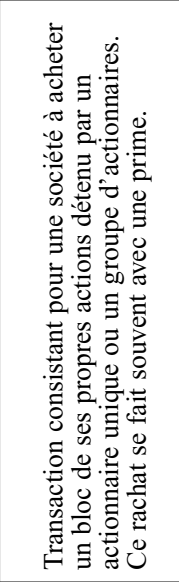 & 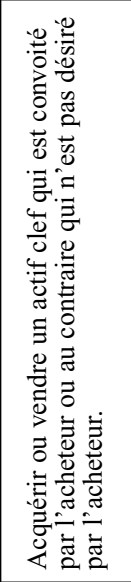 & 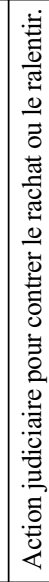 \\
\hline 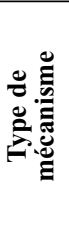 & 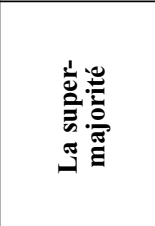 & 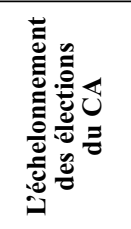 & & 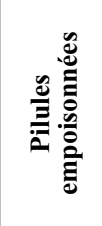 & 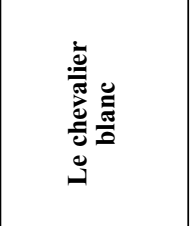 & 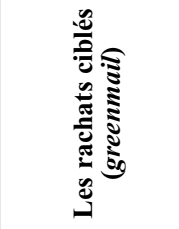 & 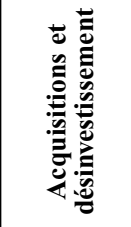 & 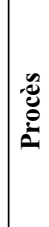 \\
\hline
\end{tabular}




\section{CONCLUSION}

Bien que les techniques décrites dans cette synthèse fassent partie des outils le plus souvent utilisés pour faire échouer une acquisition, il existe bien d'autres méthodes pour tenter de mettre en échec un acquéreur. La société peut par exemple refondre son capital en deux types d'actions (ce que les anglophones nomment la dual class recapitalization) ou également modifier sa politique de dividendes afin de récompenser la fidélité des actionnaires ou même proposer des dispositions de rémunération pour les actionnaires qui n'apportent pas leurs titres à $1^{1}$ offre ${ }^{6}$. Il est d'ailleurs important de noter que ces dispositifs ne sont pas mutuellement exclusifs et qu'au contraire, ils sont bien souvent associés.

Les résultats de cette synthèse confirment plutôt l'idée selon laquelle les moyens de défense anti-acquisition profiteraient essentiellement aux dirigeants. La théorie de l'enracinement voit ainsi son interprétation validée: les dirigeants utilisent les mécanismes de défense anti-acquisition pour se soustraire à la discipline imposée par le marché pour le contrôle des sociétés. Ils font ainsi primer leur intérêt personnel sur l'intérêt des actionnaires. Si l'impact des différents mécanismes de défense sur la richesse des actionnaires varie selon le dispositif utilisé et s'il existe des circonstances où l'utilisation de tel ou tel moyen de défense est cohérente avec l'intérêt des actionnaires, il apparaît toutefois, que l'adoption de mécanisme de défense semble, globalement, destructrice de richesse pour les actionnaires.

C'est pourquoi, en dépit de l'importante utilisation de ces dispositifs par les sociétés ${ }^{7}$, les actionnaires tentent de s'organiser pour faire disparaître les défenses antiacquisitions des sociétés. Selon Faleye (2006), le 23 avril 2003, $85 \%$ des actionnaires représentés à la réunion annuelle de Baker Hughes ont voté en faveur d'une proposition demandant l'annulation des élections échelonnées du conseil d'administration et l'élection des dirigeants chaque année. Selon Investor Responsability Resaerch Center, 51 propositions d'actionnaires d'annulation d'échelonnement des conseils d'administration ont été réalisées en 2002 et 56 ont été réalisées en 2003. De même, Akyol et Carroll (2006a) montrent que la cadence des retraits de pilules empoisonnées a augmenté au cours des dernières années et que la plupart des retraits de ces dispositifs ont eu lieu après 2001. Selon Akyol et Carroll (2006b), ce résultat est dû à l'activisme des actionnaires.

6. Ceci fut le cas notamment dans le cadre de la tentative d'opposition d'Arcelor à l'offre de Mittal.

7. Ainsi, selon l'Investor Responsibility Research Center, en 1998, 59 \% des sociétés américaines ont des conseils d'administration à élections échelonnées. 


\section{Bibliographie}

Akyol A., Carroll C., Why do firms remove poison pills?, University of Alabama, Department of economics, finance, and legal studies, 2006a.

Akyol A., Carroll C., Removing poison pills: a case of shareholder activism, University of Alabama, Department of economics, finance, and legal studies, 2006b.

Albouy M., « La finance contre l'entreprise ? », Revue française de gestion, $\mathrm{n}^{\circ}$ 93, marsmai 1993, p. 29-38,

Albouy M., « Peut-on réconcilier les dirigeants d'entreprise et les marchés financiers?», Revue française de gestion, $\mathrm{n}^{\circ} 111,1996$, p. 94-101.

Ambrose B., Megginson W., "The role of asset structure, ownership structure, and takeover defenses in determining acquisition likelihood", Journal of Financial \& Quantitative Analysis, $\mathrm{n}^{\circ} 27,1992$, p. 575-589.

Baron D., "Tender offers and management resistance", Journal of Finance, ${ }^{\circ}$ 38, 1983, p. 331-343.

Bebchuk L., Cohen A., "The costs of entrenched boards", Journal of Financial Economics, $\mathrm{n}^{\circ} 78,2005$, p. 409-433.

Bebchuk L., Coates J., Subramaniam G., "The powerful antitakeover force of staggered boards: theory, evidence, and policy”, Standford Law Review, ${ }^{\circ}$ 54, 2002, p. 887-952,

Born J., Trahan E., Faria H., "Golden parachutes: incentive aligners, management entrenchers, or takeover bid signals?", Journal of Financial Research, $\mathrm{n}^{\circ}$ 16, 1993, p. 299-308,

Borokhovich K., Brunarski K., "CEO contracting and antitakeover amendments”, Journal of Finance, $\mathrm{n}^{\circ}$ 52, 1997, p. 1495-1517.

Bradley M., Wakeman M., "The wealth effects of targeted share repurchases", Journal of Financial Economics, n ${ }^{\circ} 11,1983$, p. 301-328.

Brickley J., Coles J., Terry R., "Outside directors and the adoption of poison pills", Journal of Financial Economics, ${ }^{\circ}$ 35, 1994, p. 371-390.

Buchholtz A., Ribbens B., "Role of chief executive officers in takeover resistance: effects of CEO incentives and individual characteristics", Academy of Management Journal, $\mathrm{n}^{\circ} 37$, 1994, p. 554-579.

Choi D., Kamma S., Weintrop J., "The delaware courts, poison pills, and shareholder wealth", Journal of Law, Economics \& Organization, n ${ }^{\circ}$, 1989, p. 375-393.

Comment R., Schwert G. W., "Poison or placebo? Evidence on the deterrence and wealth effects of modern antitakeover measures", Journal of Financial Economics, n 39, 1995, p. 3-43.

Dann L., DeAngelo H., "Standstill agreements, privately negotiated stock repurchases, and the market for corporate control", Journal of Financial Economics, n 11, 1983, p. 275-300.

Datta S., Iskandar-Datta M., "Takeover defenses and wealth effects on securityholders: the case of poison pill adoptions", Journal of Banking \& Finance, n² 20, 1996, p. 1231-1250. 
DeAngelo H., Rice E., “Antitakeover charter amendments and stockholder wealth", Journal of Financial Economics, n ${ }^{\circ} 11,1983$, p. 329-360.

Faleye O., "Classified boards, firm value, and managerial entrenchment", Journal of Financial Economics, $\mathrm{n}^{\circ}$ 2, 2007, p. 501-529.

Harris E., "Antitakeover measures, golden parachutes, and target firm shareholder welfare", RAND Journal of Economics, $\mathrm{n}^{\circ} 21,1990$, p. 614-625.

Holderness C., Sheehan D., "Raiders or saviours? The evidence on six controversial investors", Journal of Financial Economics, n 14, 1985, p. 555-579.

Jarrell G., "The wealth effects of litigation by targets: do interests diverge in a merge?", Journal of Law and Economics, $\mathrm{n}^{\circ} 28,1985$, p. 151-77.

Jarrell G., Poulsen A., "Shark repellents and stock prices: the effects of antitakeover amendments since 1980", Journal of Financial Economics, n ${ }^{\circ}$ 19, 1987, p. 127-168,

Jensen M., "Takeovers: their causes and consequences", Journal of Economic Perspectives, $\mathrm{n}^{\circ} 2,1988$, p. 21-48.

Klein A., Rosenfeld J., "Targeted share repurchases and top management changes", Journal of Financial Economics, ${ }^{\circ} 20,1988$ a p. 493-506.

Klein A., Rosenfeld J., "The impact of targeted share repurchases on the wealth of nonparticpating sharehiolders", Journal of Financial Research, $\mathrm{n}^{\circ} 11,1988$ b, p. 89-97.

Knoeber C., "Golden parachutes, shark repellents, and hostile tender offers", American Economic Review, n 76,1986 , p. 155-167.

Lambert R., Larcher D., "Golden parachutes, executive decision making, and shareholder wealth", Journal of Accounting \& Economics, n 7, 1985, p. 179-203.

Linn S., McConnell J., "An empirical investigation of the impact of antitakeover amendments on common stock prices", Journal of Financial Economics, ${ }^{\circ} 11,1983$, p. 361-399.,

Mahoney J., Mahoney J., "An empirical investigation of the effect of corporate charter antitakeover amendments on stockholder wealth", Strategic Management Journal, ${ }^{\circ}{ }^{14}$, 1993, p. 17-31.

Mahoney J., Sundaramurthy C., Mahoney J., "The effects of corporate antitakeover provisions on long-term investment: empirical evidence", Managerial \& Decision Economics, n 18, 1997, p. 349-365.

Malatesta P., Walkling R., "Poison pill securities: stockholder wealth, profitability, and ownership structure", Journal of Financial Economics, n 20, 1988, p. 347-376,

Mikkelson W., Ruback R., "An empirical analysis of the interfirm equity investment process", Journal of Financial Economics, n 14, 1985, p. 523-553.

Mikkelson W., Ruback R., "Targeted repurchases and common stock returns", RAND Journal of Economics, ${ }^{\circ} 22,1991$, p. 544-561.

Mogavero D., Toyne M., "The impact of golden parachutes on Fortune 500 stock returns: a reexamination of the evidence", Quarterly Journal of Business \& Economics, ${ }^{\circ} 34,1995$, p. 30-38.

Officer M., "Termination fees in mergers and acquisitions", Journal of Financial Economics, $\mathrm{n}^{\circ} 69,2003$, p. 431-467. 
Pound J., "The effects of antitakeover amendements on takeover activity: some direct evidence", Journal of Law and Economics, ${ }^{\circ} 30,1987$, p. 353-367.

Ryngaert M., "The effect of poison pill securities on shareholder wealth", Journal of Financial Economics, n $^{\circ} 20$, 1988, p. 377-417.

Scherer F., "Corporate takeovers: the efficiency arguments", Journal of Economic Perspectives, $\mathrm{n}^{\circ} 2,1988$, p. 69-82.

Schwert W., "Markup pricing in mergers and acquisitions", Journal of Financial Economics, $\mathrm{n}^{\circ}$ 41, 1996, p. 153-192.

Shleifer A., Vishny R., "Greenmail, white knights, and shareholder" interest", RAND Journal of Economics, n 17, 1986, p. 293-309.

Siegel P., Karim K., "The information content of the adoption of classified board provision", Journal of Financial \& Strategic Decisions, ${ }^{\circ}$ 11, 1998, p. 51-59.

Stein J., "Takeover threats and managerial myopia", Journal of Political Economy, n 96 , 1988, p. 61-80.

Sundaramurthy C., Rechner P., Wang W., "Governance antecedents of board entrenchment: the case of classified board provisions", Journal of Management, ${ }^{\circ} 22,1996$, p. 783-799.

Schwartz M., "The Massachusetts classified board law", Journal of Economics \& Finance, $\mathrm{n}^{\circ} 22,1998$, p. 29-36. 
